\title{
Masked hypertension and cardiovascular outcomes: an updated systematic review and meta-analysis
}

This article was published in the following Dove Press journal: Integrated Blood Pressure Control

\author{
Mohan Palla' \\ Hamidreza Saber ${ }^{\prime}$ \\ Sanjana Konda' \\ Alexandros Briasoulis ${ }^{2}$ \\ 'Division of Cardiology, Wayne State \\ University, Detroit, MI, ${ }^{2}$ Section of \\ Heart Failure and Transplant, Division \\ of Cardiovascular Diseases, University \\ of lowa Hospitals and Clinics, lowa \\ City, IA, USA
}

Background: As many as one-third of individuals with normal office blood pressure (BP) are diagnosed with masked hypertension (HTN) based on ambulatory BP measurements (ABPM). Masked HTN is associated with higher risk of sustained HTN (SH) and increased cardiovascular morbidity.

Methods: The present study was designed to systematically review cohort studies and assess the effects of masked HTN compared to normotension and SH on cardiovascular events and all-cause mortality. We systematically searched the electronic databases, such as MEDLINE, PubMed, Embase, and Cochrane for prospective cohort studies, which evaluated participants with office and ambulatory and/or home BP.

Results: We included nine studies with a total number of 14729 participants (11245 normotensives, 3484 participants with masked HTN, 1984 participants with white-coat HTN, and 5143 participants with $\mathrm{SH}$ ) with a mean age of 58 years and follow-up of 9.5 years. Individuals with masked HTN had significantly increased rates of cardiovascular events and all-cause mortality than normotensives and white-coat HTN and had lower rates of cardiovascular events than those with $\mathrm{SH}$ (odds ratio $0.61,95 \%$ confidence interval $0.42-0.89 ; P=0.010 ; I^{2}=84 \%$ ). Among patients on antihypertensive treatment, masked HTN was associated with higher rates of cardiovascular events than in those with normotension and white-coat HTN and similar rates of cardiovascular events in those with treated SH.

Conclusion: Prompt screening of high-risk individuals with home BP measurements and ABPM, the diagnosis of masked HTN, and the initiation of treatment, may mitigate the adverse cardiovascular effects of masked HTN.

Keywords: masked hypertension, cardiovascular outcomes, meta-analysis

\section{Introduction}

Prevalence rates of hypertension (HTN) in the US adult population have increased remarkably over the past two decades but appear to have remained unchanged over the past 10 years, according to data from the National Health and Nutrition Examination Survey (NHANES). HTN contributes to one out of every seven deaths in the US and to nearly half of all cardiovascular disease-related deaths, including stroke. Despite the success of drug therapy in treating HTN and reducing associated adverse cardiovascular effects, the percentage of patients achieving adequate blood pressure (BP) control worldwide remains unacceptably low. Recent data suggest an improvement in the treatment and control of HTN; however, $\sim 48 \%$ of those treated are not at a BP goal of $<140 / 90 \mathrm{mmHg}$ in the US. ${ }^{1}$ These numbers are even higher among
Correspondence: Alexandros Briasoulis Section of Heart Failure and Transplant, Division of Cardiovascular Diseases, University of lowa Hospitals and Clinics, 200 Hawkins Drive, lowa City, IA 52242, USA

Tel + I 3196788418

Fax + I 3193536343

Email alexbriasoulis@gmail.com 
hypertensive patients in Europe. ${ }^{2}$ Importantly, about one-third of hypertensives whose BP levels appear well controlled according to clinic BP measurements have high BP outside the doctor's office. ${ }^{3,4}$ The frequency of ambulatory HTN is even higher among patients with chronic kidney disease. ${ }^{5}$ This phenomenon is called masked HTN or isolated ambulatory HTN and is defined as clinic BP $<140 / 90 \mathrm{mmHg}$ and daytime ambulatory $\mathrm{BP} \geq 135 / 85 \mathrm{mmHg}$. An analysis of Dallas Heart Study suggested that in a multiethnic US population, masked HTN was independently associated with increased aortic stiffness, renal injury, and incident cardiovascular events and its prevalence was $17.8 \%{ }^{6}$ Furthermore, a prospective analysis of the Jackson Heart study cohort of African Americans showed an incidence of masked HTN of $52 \%$ and an association with cardiovascular events but not all-cause mortality. ${ }^{7}$ Moreover, masked HTN was found to be associated with symptoms of end-organ damage such as left ventricular hypertrophy, increased pulse wave velocity, and carotid intima and media thickness. ${ }^{4}$

To further explore the outcomes of masked HTN both in treated and untreated groups of patient populations, we performed an updated meta-analysis.

\section{Methods}

\section{Search strategy}

Systematic electronic search was performed on MEDLINE (PubMed interface), Embase, and Cochrane Central Register of Controlled Trials (CENTRAL) with no language limitations. We used the MESH terms "masked hypertension" and "white coat hypertension". Two reviewers (MP and AB) independently screened titles and abstracts based on inclusion and exclusion criteria. After eliminating irrelevant articles, full-text reports were reviewed. Subsequently, we hand searched all included studies until no further relevant studies were identified. Disagreements between the two reviewers were resolved by the third reviewer. Finally, a total of nine studies have been identified. The electronic search was last updated on September 20, 2017.

\section{Study selection}

We included randomized clinical trials, prospective and retrospective observational studies published as original articles in peer-reviewed scientific journals. The population studied was patients who were treated for masked HTN, white-coat HTN, normotension, or sustained HTN (SH). We excluded nonhuman studies, incorrect comparator studies, single-arm noncomparative studies, and review articles. We did not restrict eligibility based on study outcomes.

\section{Data extraction and quality}

The data were independently extracted by two authors (MP and $\mathrm{AB}$ ) using standardized protocol and data extraction form. Disagreements were resolved by discussion with the third reviewer, and consensus was reached after discussion. We extracted data on outcome measures, study characteristics, such as study design, year of study, study population, sample size, and follow-up duration, and patient characteristics, such as baseline demographic and clinical characteristics.

\section{Assessment of outcomes}

Our primary outcome measure was composite cardiovascular events. Secondary outcome measures were all-cause mortality and stroke.

\section{Risk of bias}

Cochrane's risk of bias tool has been used in order to assess the individual risk of bias of each study. ${ }^{19-21}$ Two authors (MP and $\mathrm{AB}$ ) independently assessed the risk of bias and quality of studies in each eligible trial. The criteria used for the risk of bias assessment were adequate sequence generation, allocation concealment, blinding of participants, research personnel and outcome assessors, personnel and outcome assessors, incomplete outcome data, and selective outcome reporting. Low-quality studies had two or more quality assessment criteria qualified as high or unclear risk of bias.

\section{Data analysis, summary measures, and synthesis of results}

Systematic review and meta-analysis were done in compliance with the Cochrane Collaboration and the Preferred Reporting Items for Systematic Reviews and Meta-Analyses (PRISMA) Statement. ${ }^{19-21}$ Meta-analyses were performed by using the Review Manager (RevMan) Version 5.3 (Nordic Cochrane Centre, The Cochrane Collaboration, 2012, Copenhagen, Denmark). Chi-square test of heterogeneity and $I^{2}$ statistic of inconsistency were used to assess the heterogeneity between studies. $I^{2}$ values of 25,50 , and $75 \%$ were considered as low, moderate, and high heterogeneities, respectively. ${ }^{22}$ A significant heterogeneity was considered if $P<0.05$ or $I^{2}$ statistics $>25 \%$. Pooled effect of intervention was measured using odds ratio (OR) or standardized mean difference (SMD) with $95 \%$ confidence interval (CI). Mantel-Haenszel fixed-effects model was used to estimate the pooled effect measure if the heterogeneity is $<25 \%$. In case of significant heterogeneity between studies, DerSimonian and Laird ${ }^{23}$ randomeffects model was used. Reported values are two tailed, 
and hypothesis testing results were considered statistically significant at $P<0.05$. Sensitivity analyses were performed by eliminating each study at a time to assess the influence of any included study on the results and the robustness of results. Significant heterogeneity between the studies was further explored using subgroup analyses. The small study effect, including publication bias, was tested using the visual estimation of funnel plot for asymmetry and Egger's regression test. ${ }^{24,25}$ If visual asymmetry funnel plot was found, then nonparametric trim and fill method of Duvall and Tweedie was performed to add studies that appeared to be missing. ${ }^{26}$

\section{Results}

\section{Study characteristics}

Electronic search of scientific literature identified 1553 articles. After deduplication, screening of titles and abstracts, and full-text review based on inclusion and exclusion criteria, nine studies were identified and included in the meta-analysis. ${ }^{6,7,16,27-32}$ The characteristics of each study are shown in Table 1.

\section{Patient characteristics}

A total number of 14729 participants (11245 normotensives, 3484 participants with masked HTN, 1984 participants with white-coat HTN, and 5143 participants with SH) were included in the meta-analysis. The mean age of the study population was 58 years. Other baseline demographics and clinical characteristics are shown in Table 2 . The mean follow-up duration was 9.5 years. Clinic and ambulatory mean BP measurements are shown in Table 3.

\section{Masked HTN versus normotension}

\section{Composite cardiovascular events}

Composite cardiovascular events occurred in $12.3 \%$ of patients with masked HTN and $5.1 \%$ of patients with normotension. The frequency of cardiovascular events was significantly higher in patients with masked HTN (OR 2.91, 95\% CI 2.54-3.33; $P<0.00001 ; I^{2}=17 \%$, Figure 1A). No significant heterogeneity was seen among studies.

\section{All-cause mortality}

All-cause death occurred in $15.8 \%$ of patients with masked HTN and $7.8 \%$ of patients with normotension. The risk of all-cause mortality was significantly higher in patients with masked HTN (OR 2.65, 95\% CI 2.18-3.23; $P<0.00001$; $I^{2}=0 \%$, Figure $1 \mathrm{~B}$ ) without significant heterogeneity among studies.

\section{Masked HTN versus white-coat HTN Composite cardiovascular events}

Composite cardiovascular events were encountered in $12.8 \%$ of patients with masked HTN and $10.8 \%$ of patients with white-coat HTN, and the risk of cardiovascular events was significantly higher in patients with masked HTN (OR $1.38,95 \%$ CI 1.04-1.83; $P=0.02 ; I^{2}=41 \%$, Figure 2 A) with significant heterogeneity among studies.

\section{All-cause mortality}

The rates of all-cause mortality were significantly elevated in patients with masked HTN (OR 1.71, 95\% CI 1.34-2.19; $P<0.0001 ; P^{2}=0 \%$, Figure $2 \mathrm{~B}$ ) without significant heterogeneity among studies.

\section{Masked HTN versus SH \\ Composite cardiovascular events}

Composite cardiovascular events occurred in $12.7 \%$ of patients with masked HTN and $19.3 \%$ of patients with SH. We found significantly lower risk of cardiovascular events in patients with masked HTN compared with patients with $\mathrm{SH}$ (OR 0.61, 95\% CI 0.42-0.89; $P=0.010 ; I^{2}=84 \%$, Figure 3 A).

Table I Study characteristics

\begin{tabular}{|c|c|c|c|c|c|c|}
\hline \multirow[t]{2}{*}{ Study } & \multirow[t]{2}{*}{ Study design } & \multicolumn{4}{|c|}{ Sample size } & \multirow{2}{*}{$\begin{array}{l}\text { Mean follow- } \\
\text { up (years) }\end{array}$} \\
\hline & & $\begin{array}{l}\text { Masked } \\
\text { HTN }\end{array}$ & Normotension & $\begin{array}{l}\text { White-coat } \\
\text { HTN }\end{array}$ & $\begin{array}{l}\text { Sustained } \\
\text { HTN }\end{array}$ & \\
\hline Björklund et al (2003) $)^{27}$ & Prospective observational study & 82 & 188 & & 308 & 5.9 \\
\hline Fagard et al $(2005)^{28}$ & Prospective observational study & 31 & 136 & 87 & 105 & 10.9 \\
\hline Mancia et al $(2006)^{29}$ & Prospective observational study & 184 & 909 & 242 & 528 & 12.3 \\
\hline Hansen et al $(2006)^{30}$ & Prospective observational study & 211 & 859 & 159 & 471 & 12.8 \\
\hline Pierdomenico et al $(2008)^{31}$ & Prospective observational study & 120 & 471 & & & 6.6 \\
\hline Stergiou et al $(2014)^{16}$ & Prospective observational study & 636 & 3312 & 925 & 1585 & 8.3 \\
\hline Asayama et al $(2014)^{32}$ & Prospective observational study & 1612 & 4176 & 515 & 1934 & II.I \\
\hline Booth et al $(2016)^{7}$ & Prospective observational study & 385 & 353 & & & 8.5 \\
\hline Tientcheu et al $(2017)^{6}$ & Prospective observational study & 256 & 865 & 56 & 212 & 9.5 \\
\hline
\end{tabular}

Abbreviation: HTN, hypertension. 
Table 2 Baseline clinical characteristics

\begin{tabular}{|c|c|c|c|c|c|c|c|c|}
\hline \multirow[t]{2}{*}{ Study } & \multicolumn{4}{|c|}{ Age (mean, years) } & \multicolumn{4}{|c|}{ Males (\%) } \\
\hline & $\begin{array}{l}\text { Masked } \\
\text { HTN }\end{array}$ & $\begin{array}{l}\text { Normo- } \\
\text { tension }\end{array}$ & $\begin{array}{l}\text { White- } \\
\text { coat } \\
\text { HTN }\end{array}$ & $\begin{array}{l}\text { Sustained } \\
\text { HTN }\end{array}$ & $\begin{array}{l}\text { Masked } \\
\text { HTN }\end{array}$ & $\begin{array}{l}\text { Normo- } \\
\text { tension }\end{array}$ & $\begin{array}{l}\text { White- } \\
\text { coat } \\
\text { HTN }\end{array}$ & $\begin{array}{l}\text { Sustained } \\
\text { HTN }\end{array}$ \\
\hline Björklund et $\mathrm{al}^{27}$ & 70 & 70 & & 70 & 100 & 100 & & 100 \\
\hline Fagard et $\mathrm{al}^{28}$ & 68 & 69 & 70 & 72 & 55 & 48 & 28 & 35 \\
\hline Mancia et $\mathrm{al}^{29}$ & 53 & 44 & 52 & 60 & 68 & 43 & 53 & 58 \\
\hline Hansen et $\mathrm{al}^{30}$ & 55 & 53 & 60 & 60 & 63 & 45 & 41 & 63 \\
\hline Pierdomenico et $\mathrm{al}^{31}$ & 50 & 50 & & & 57 & 44 & & \\
\hline Stergiou et al $^{16}$ & 65 & 60 & 62 & 66 & 54 & 37 & 43 & 46 \\
\hline Asayama et $\mathrm{al}^{32}$ & 53 & 46 & 59 & 61 & 63 & 45 & 54 & 67 \\
\hline Booth et $\mathrm{al}^{7}$ & 60 & 57 & & & 37 & 21 & & \\
\hline Tientcheu et $\mathrm{al}^{6}$ & 47 & 40 & 49 & 50 & 47 & 44 & 42 & 46 \\
\hline
\end{tabular}

Abbreviations: BMI, body mass index; DM, diabetes mellitus; HTN, hypertension.

However, significant heterogeneity was presented among the included studies.

\section{All-cause mortality}

We did not identify significant differences in the all-cause mortality rates between patients with masked and SH (OR $1.00,95 \%$ CI $0.44-2.26 ; P=1.0 ; P^{2}=90 \%$, Figure 3B). Significant heterogeneity was found among the included studies.

\section{Patients without treatment \\ Masked HTN versus normotension - untreated patients}

Composite cardiovascular events

We observed significantly higher rates of cardiovascular events in patients with masked HTN (OR 3.10, 95\% CI $2.50-3.83 ; P<0.00001 ; P^{2}=4 \%$, Figure $4 \mathrm{~A}$ ) without significant heterogeneity among studies.

\section{All-cause mortality}

All-cause death occurred in $10.3 \%$ of patients with masked HTN and $3.5 \%$ of patients with normotension. The risk of all-cause mortality was significantly higher in patients with masked HTN (OR 3.12, 95\% CI 2.24-4.35; $P<0.00001$; $P=28 \%$, Figure 4B) without significant heterogeneity among studies.

\section{Masked HTN versus white-coat HTN - untreated patients \\ Composite cardiovascular events}

Composite cardiovascular events occurred in $11.9 \%$ of patients with masked HTN and $9.6 \%$ of patients with whitecoat HTN with significantly higher risk of cardiovascular events in patients with masked HTN (OR 1.33, 95\% CI
$1.01-1.75 ; P=0.04 ; I^{2}=3 \%$, Figure $5 \mathrm{~A}$ ) without significant heterogeneity among studies.

\section{All-cause mortality}

All-cause mortality occurred in $10.7 \%$ of patients with masked HTN and $8.5 \%$ of patients with white-coat HTN. The risk of all-cause mortality was nonsignificantly higher in patients with masked HTN (OR 1.62, 95\% CI 1.01-2.60; $P=0.05 ; P=41 \%$, Figure 5B).

\section{Masked HTN versus SH - untreated patients \\ Composite cardiovascular events}

Composite cardiovascular events were reported in $11.9 \%$ of patients with masked HTN and $16.7 \%$ of patients with SH. The risk of cardiovascular events was significantly lower in patients with masked HTN compared with patients with $\mathrm{SH}$ (OR $0.68,95 \%$ CI $0.53-0.86 ; P=0.001 ; I^{2}=20 \%$, Figure 6 A) without significant heterogeneity among studies.

\section{All-cause mortality}

All-cause mortality occurred in $10.7 \%$ of patients with masked HTN and $12.6 \%$ of patients with SH. No significant difference was found in the all-cause mortality rates between patients with masked and SH (OR 0.88, 95\% CI 0.33-2.37; $P=0.80 ; I^{2}=89 \%$, Figure $\left.6 \mathrm{~B}\right)$.

\section{Patients on treatment}

\section{Masked HTN versus normotension - treated} patients

\section{Composite cardiovascular events}

Composite cardiovascular events were found in $27.9 \%$ of patients with masked HTN and $15.5 \%$ of patients with normotension with significantly higher rates of cardiovascular 


\begin{tabular}{|c|c|c|c|c|c|c|c|c|c|c|c|}
\hline \multicolumn{4}{|c|}{ BMI (mean, kg/m²) } & \multicolumn{4}{|c|}{ Smokers (\%) } & \multicolumn{4}{|l|}{ DM (\%) } \\
\hline $\begin{array}{l}\text { Masked } \\
\text { HTN }\end{array}$ & $\begin{array}{l}\text { Normo- } \\
\text { tension }\end{array}$ & $\begin{array}{l}\text { White- } \\
\text { coat } \\
\text { HTN }\end{array}$ & $\begin{array}{l}\text { Sustained } \\
\text { HTN }\end{array}$ & $\begin{array}{l}\text { Masked } \\
\text { HTN }\end{array}$ & $\begin{array}{l}\text { Normo- } \\
\text { tension }\end{array}$ & $\begin{array}{l}\text { White- } \\
\text { coat } \\
\text { HTN }\end{array}$ & $\begin{array}{l}\text { Sustained } \\
\text { HTN }\end{array}$ & $\begin{array}{l}\text { Masked } \\
\text { HTN }\end{array}$ & $\begin{array}{l}\text { Normo- } \\
\text { tension }\end{array}$ & $\begin{array}{l}\text { White- } \\
\text { coat } \\
\text { HTN }\end{array}$ & $\begin{array}{l}\text { Sustained } \\
\text { HTN }\end{array}$ \\
\hline 26 & 25 & & 26 & 22 & 21 & & 22 & 5 & 6 & & 15 \\
\hline 28 & 27 & 27 & 28 & 29 & 26 & 8 & 12 & 0 & 4 & 10 & 12 \\
\hline 26 & 24 & 27 & 28 & 42 & 27 & 24 & 21 & & & & \\
\hline 26 & 24 & 26 & 27 & 52 & 50 & 22 & 38 & 3 & 1 & 1 & 4 \\
\hline 26 & 26 & & & 30 & 19 & & & & & & \\
\hline 26 & 24 & 25 & 27 & 26 & 17 & 15 & 18 & 16 & 8 & 10 & 13 \\
\hline 26 & 24 & 26 & 27 & 37 & 31 & 19 & 27 & 7 & 4 & 8 & 9 \\
\hline 31 & 31 & & & 12 & 7 & & & 31 & 17 & & \\
\hline 31 & 28 & 31 & 32 & 30 & 26 & 27 & 32 & 17 & 7 & 21 & 21 \\
\hline
\end{tabular}

events in patients with masked HTN (OR 2.03, 95\% CI 1.52-2.72; $P<0.00001 ; I^{2}=0 \%$, Figure 7A). No significant heterogeneity was observed between the studies.

\section{All-cause mortality}

All-cause mortality occurred in $15.2 \%$ of patients with masked HTN and $10.4 \%$ of patients with normotension. The risk of all-cause mortality was significantly higher in patients with masked HTN (OR 1.44, 95\% CI 1.03-2.01; $P=0.03 ; P^{2}=0 \%$, Figure 7B) without significant heterogeneity among studies.

\section{Masked HTN versus white-coat HTN - treated patients \\ Composite cardiovascular events}

Composite cardiovascular events were reported in $27.9 \%$ of patients with masked HTN and 19.4\% of patients with white-coat HTN with significantly higher risk of cardiovascular events in patients with masked HTN (OR 1.64, 95\% CI $1.20-2.25 ; P=0.002 ; P^{2}=0 \%$, Figure $8 \mathrm{~A}$ ). No significant heterogeneity was found between the studies.

\section{All-cause mortality}

All-cause mortality occurred in $21.0 \%$ of patients with masked HTN and $17.3 \%$ of patients with white-coat HTN. No significant difference was found in the rates of all-cause mortality between the patients with masked and white-coat HTN (OR 1.03, 95\% CI 0.55-1.90; $P=0.94 ; I^{2}=39 \%$, Figure 8B).

\section{Masked HTN versus SH - treated patients Composite cardiovascular events}

We did not find significant differences in the risk of cardiovascular events between patients with masked and SH (OR
$1.08,95 \%$ CI $0.71-1.65 ; P=0.72 ; I^{2}=45 \%$, Figure 9A) with significant heterogeneity among studies.

\section{All-cause mortality}

All-cause death occurred in $21.0 \%$ of patients with masked HTN and $17.1 \%$ of patients with SH without significant differences in the risk of all-cause mortality between the patients with masked and SH (OR 0.92, 95\% CI 0.26-3.22; $P=0.90 ; I^{2}=85 \%$, Figure 9B). Significant heterogeneity was observed between studies.

\section{Assessment of risk of bias}

Cochrane's risk of bias tool suggested low-to-moderate risk of bias in all studies. Assessment of individual components of Cochrane's risk of bias tool in all trials showed low risk of bias in case of sequence generation, blinding of outcome assessment, incomplete outcome data, and selective outcome reporting.

\section{Discussion}

The salient findings of our meta-analysis can be summarized as follows: 1) the composite cardiovascular events and allcause mortality were significantly higher in patients with masked HTN compared with patients with normotension and white-coat HTN; 2) composite cardiovascular events were significantly lower in patients with masked HTN compared with patients with SH; however, no significant difference was seen in all-cause mortality between the two groups; 3) similar results were observed in the sub-group analysis of patients who are untreated; and 4) in the subgroup of patients who received antihypertensive treatment, no significant difference was observed in composite cardiovascular events and all-cause mortality between the patients 


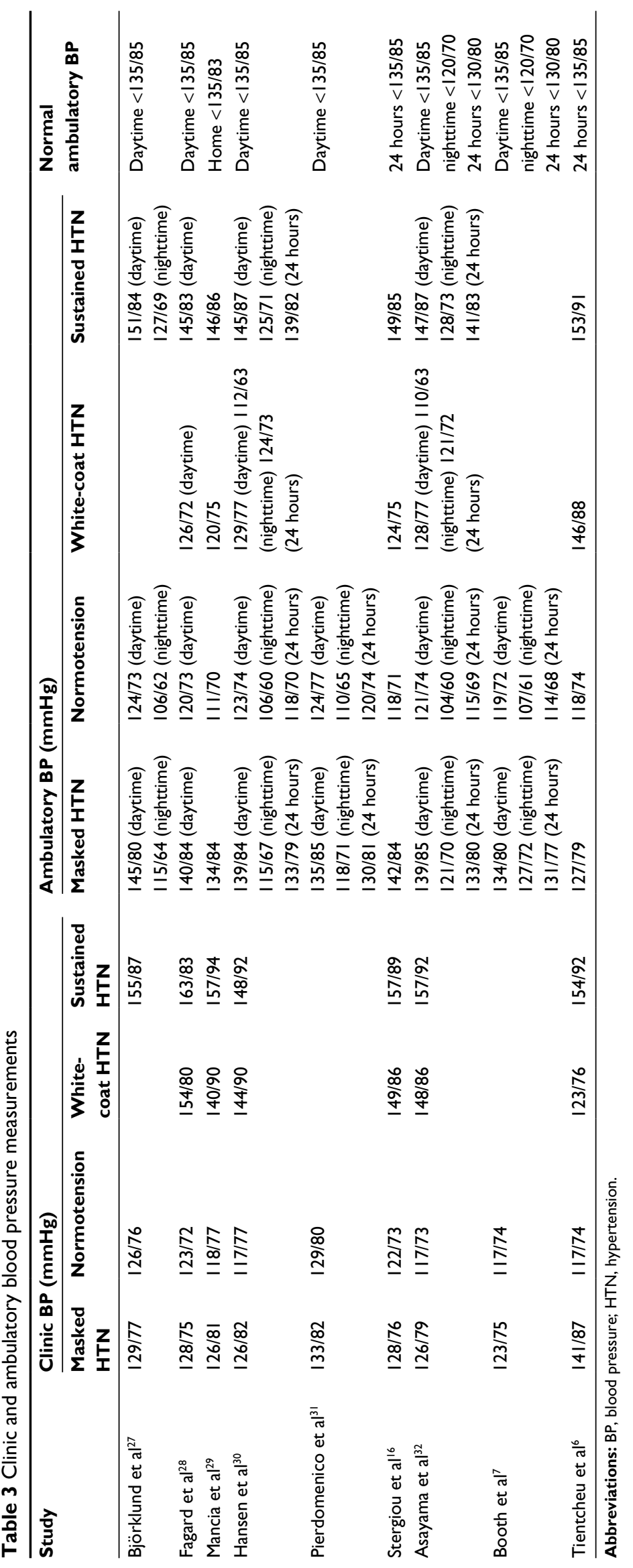


A

Composite cardiovascular events: masked HTN versus normotension

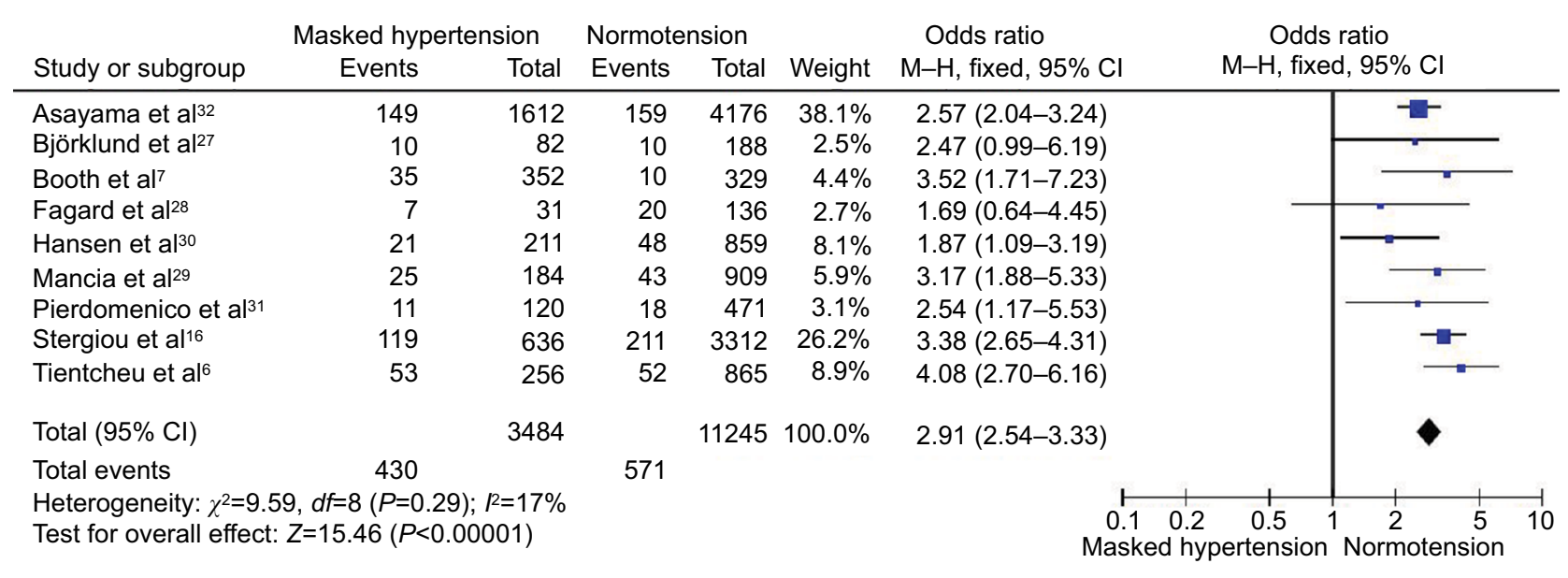

B

Mortality: masked HTN versus normotension

\begin{tabular}{|c|c|c|c|c|c|c|c|c|}
\hline \multirow[b]{2}{*}{ Study or subgroup } & \multicolumn{2}{|c|}{ Masked hypertension } & \multicolumn{2}{|c|}{ Normotension } & \multirow[b]{2}{*}{ Weight } & \multirow{2}{*}{$\begin{array}{l}\text { Odds ratio } \\
\mathrm{M}-\mathrm{H} \text {, fixed, } 95 \% \mathrm{Cl}\end{array}$} & \multirow{2}{*}{\multicolumn{2}{|c|}{$\begin{array}{c}\text { Odds ratio } \\
\mathrm{M}-\mathrm{H} \text {, fixed, } 95 \% \mathrm{Cl}\end{array}$}} \\
\hline & Events & Total & Events & Total & & & & \\
\hline Booth et al ${ }^{7}$ & 29 & 385 & 15 & 353 & $14.0 \%$ & $1.84(0.97-3.48)$ & & \\
\hline Mancia et $\mathrm{al}^{29}$ & 25 & 184 & 43 & 909 & $12.1 \%$ & $3.17(1.88-5.33)$ & & \\
\hline Stergiou et al ${ }^{16}$ & 136 & 636 & 301 & 3312 & $73.9 \%$ & $2.72(2.18-3.40)$ & & \\
\hline Total $(95 \% \mathrm{Cl})$ & & 1205 & & 4574 & $100.0 \%$ & $2.65(2.18-3.23)$ & & \\
\hline Total events & 190 & & 359 & & & & & \\
\hline $\begin{array}{l}\text { Heterogeneity: } \chi^{2}=1 \\
\text { Test for overall effec }\end{array}$ & $\begin{array}{l}6, d f=2(P=0 . \\
Z=9.67(P<0\end{array}$ & $\begin{array}{l}12=0 \% \\
1)\end{array}$ & & & & Mas & $\begin{array}{c}1 \\
0.5 \\
\text { pertension }\end{array}$ & $\begin{array}{c}1 \\
\text { Normoter }\end{array}$ \\
\hline
\end{tabular}

Figure I Masked HTN versus normotension - whole cohort.

Notes: (A) Composite cardiovascular events. (B) All-cause mortality.

Abbreviations: $\mathrm{Cl}$, confidence interval; $\mathrm{HTN}$, hypertension; $\mathrm{M}-\mathrm{H}$, Mantel-Haenszel.

A

Composite cardiovascular events: masked HTN versus white-coat HTN

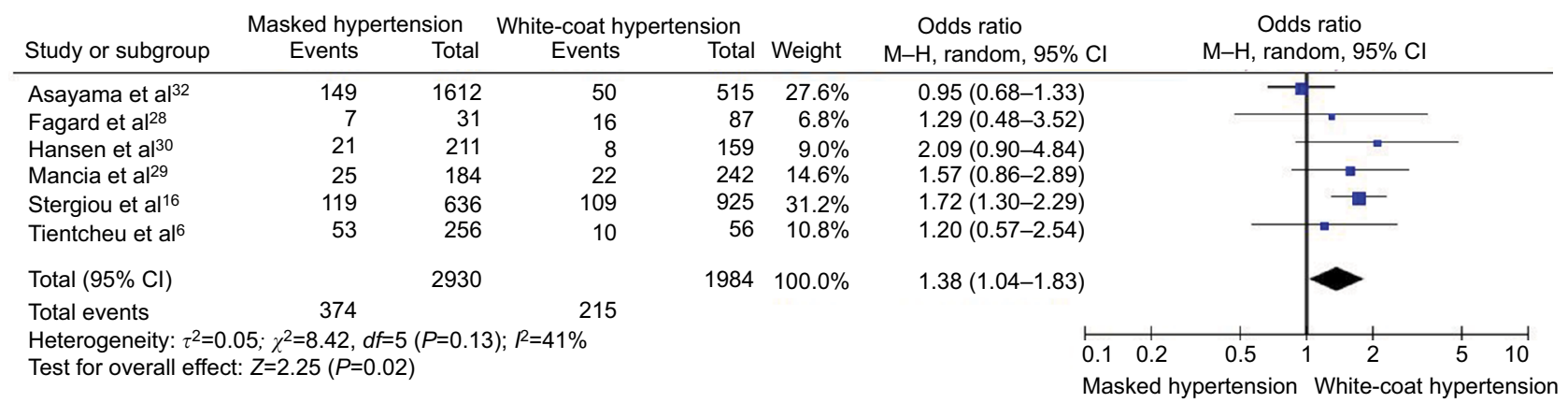

B

Mortality: masked HTN versus white-coat HTN

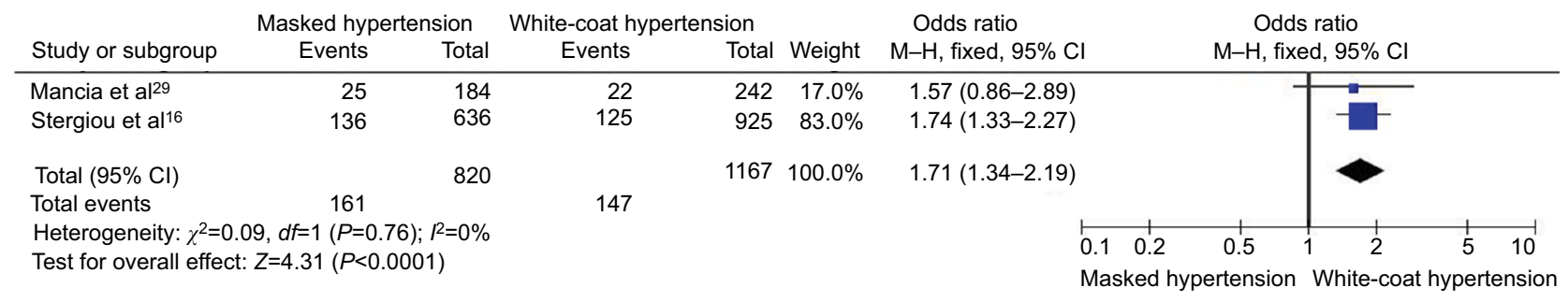

Figure 2 Masked HTN versus white-coat HTN - whole cohort.

Notes: (A) Composite cardiovascular events. (B) All-cause mortality.

Abbreviations: $\mathrm{Cl}$, confidence interval; HTN, hypertension; $\mathrm{M}-\mathrm{H}$, Mantel-Haenszel. 
A

Composite cardiovascular events: masked HTN versus sustained HTN

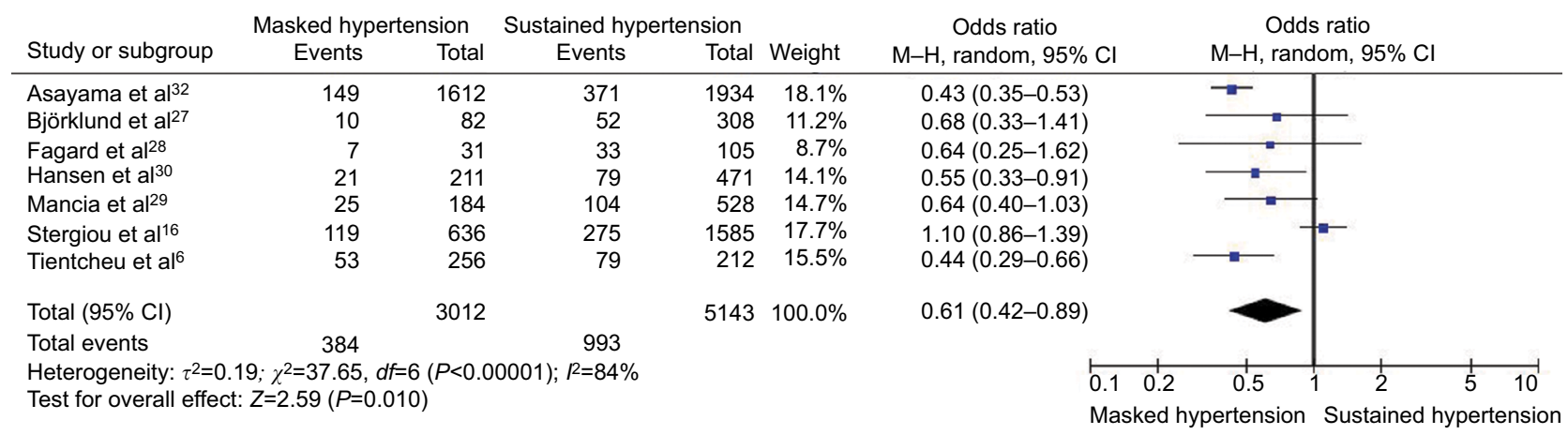

B

Mortality: masked HTN versus sustained HTN

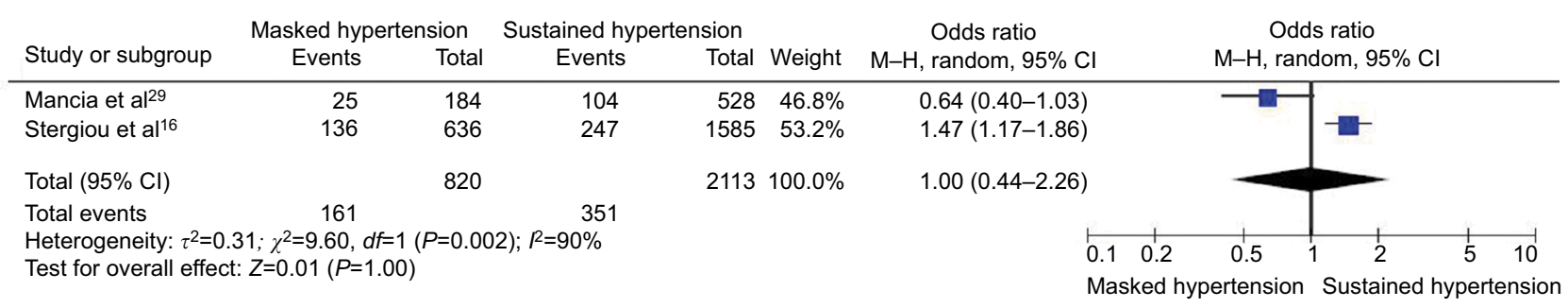

Figure 3 Masked HTN versus sustained HTN - whole cohort.

Notes: (A) Composite cardiovascular events. (B) All-cause mortality.

Abbreviations: $\mathrm{Cl}$, confidence interval; $\mathrm{HTN}$, hypertension; $\mathrm{M}-\mathrm{H}$, Mantel-Haenszel.

A

Composite cardiovascular events: masked HTN versus normotension

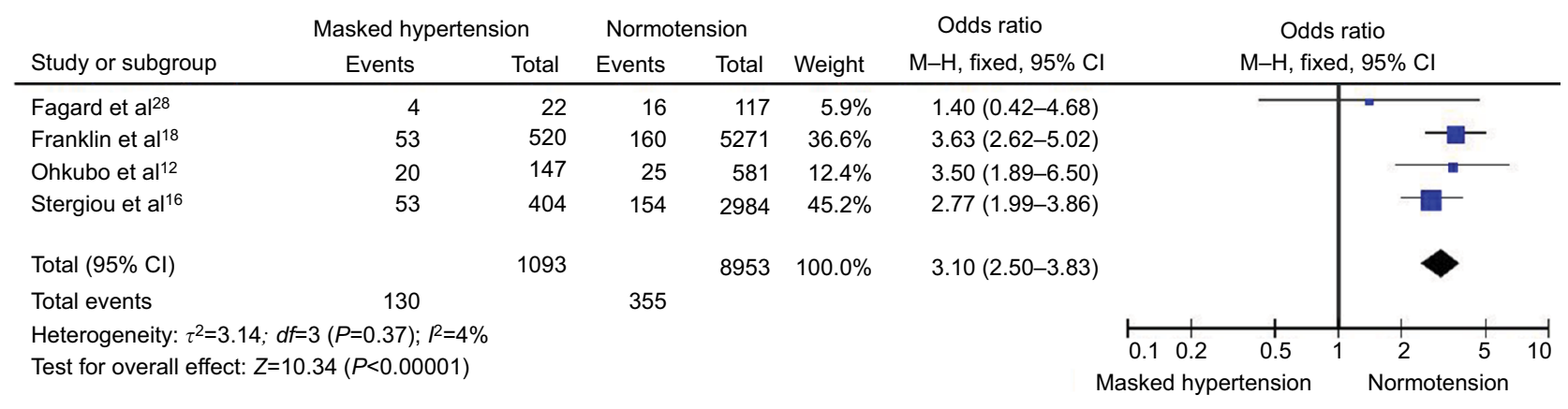

B

Mortality: masked HTN versus normotension

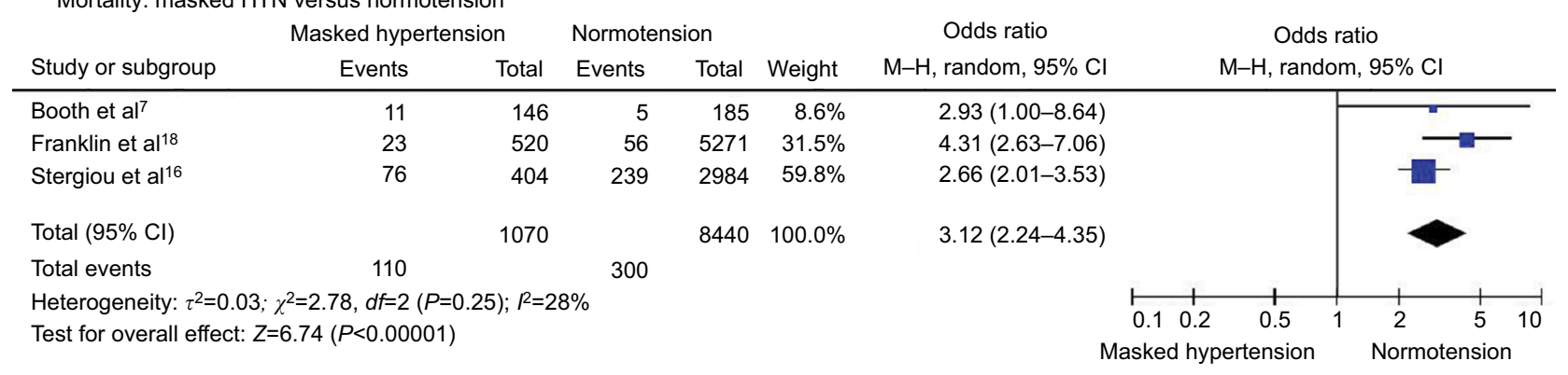

Figure 4 Masked HTN versus normotension - untreated.

Notes: (A) Composite cardiovascular events. (B) All-cause mortality.

Abbreviations: $\mathrm{Cl}$, confidence interval; $\mathrm{HTN}$, hypertension; $\mathrm{M}-\mathrm{H}$, Mantel-Haenszel. 
A

Composite cardiovascular events: masked HTN versus white-coat HTN

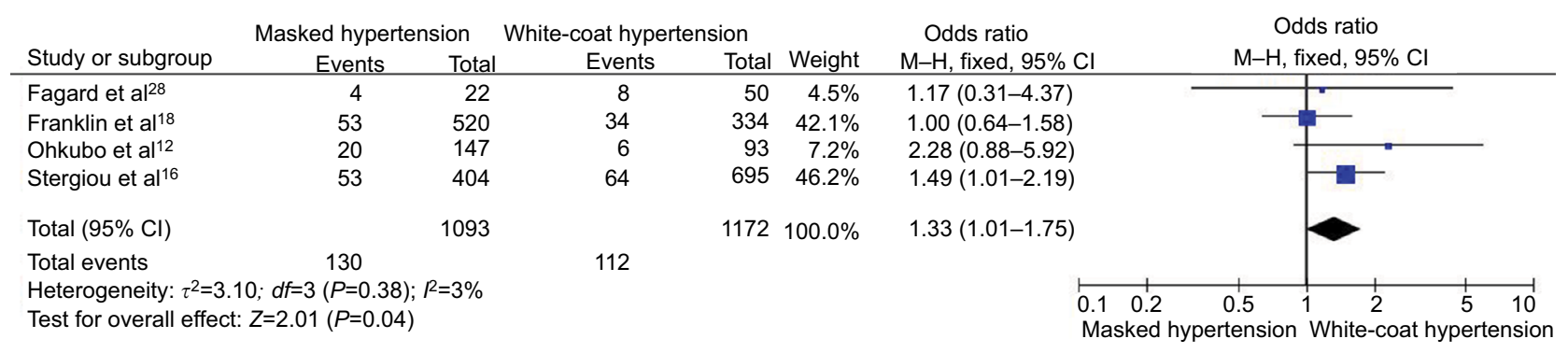

B

Mortality: masked HTN versus white-coat HTN

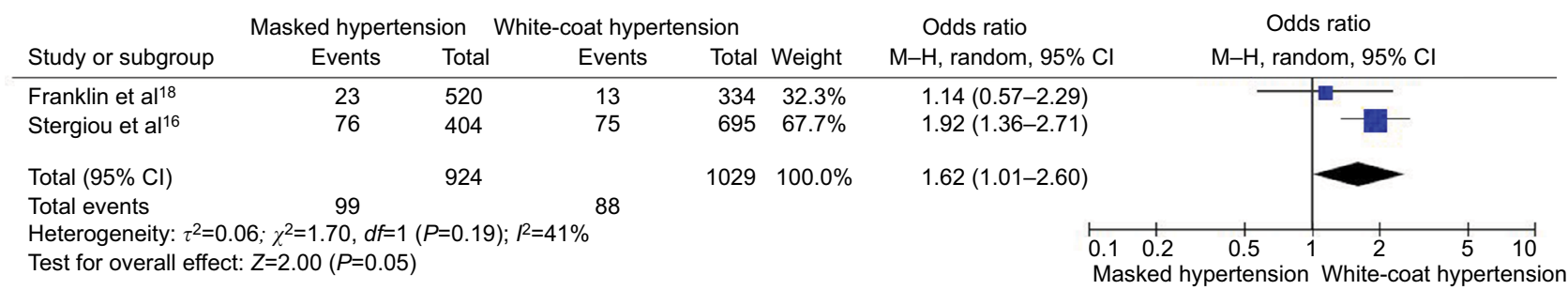

Figure 5 Masked HTN versus white-coat HTN - untreated.

Notes: (A) Composite cardiovascular events. (B) All-cause mortality.

Abbreviations: $\mathrm{Cl}$, confidence interval; HTN, hypertension; $\mathrm{M}-\mathrm{H}$, Mantel-Haenszel.

A

Composite cardiovascular events: masked HTN versus sustained HTN

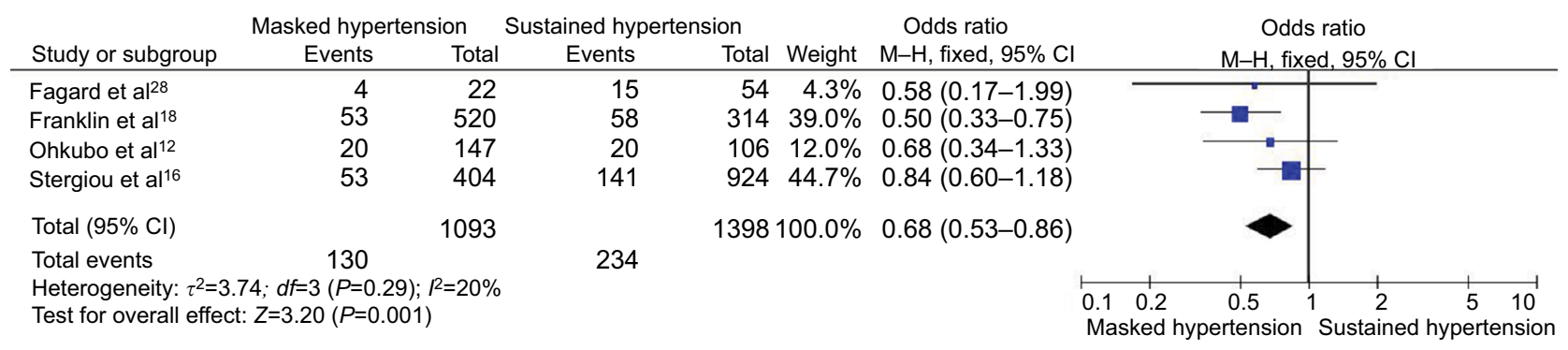

B

Mortality: masked HTN versus sustained HTN

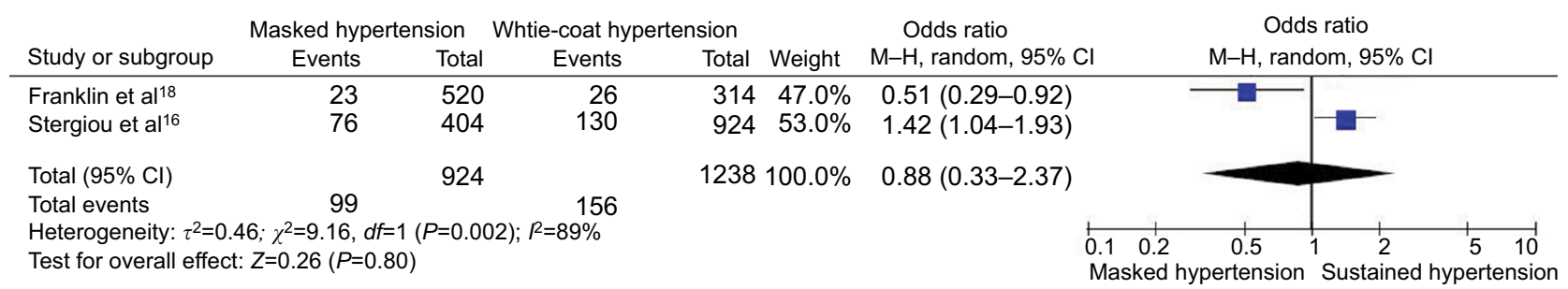

Figure 6 Masked HTN versus sustained HTN - untreated.

Notes: (A) Composite cardiovascular events. (B) All-cause mortality.

Abbreviations: $\mathrm{Cl}$, confidence interval; HTN, hypertension; $\mathrm{M}-\mathrm{H}$, Mantel-Haenszel.

with masked and SH. Among treated patients, masked HTN was associated with higher rates of cardiovascular events compared with normotension and white-coat HTN.

Early identification and diagnosis of masked HTN with 24-hour ambulatory BP measurements (ABPM) and home BP measurements (HBPM) are important. Although HMBP lacks night-time measurement and may misclassify some masked HTN patients as normotensives, ABPM may have limited tolerability, affordability, and availability particularly in the US. Despite the limited sensitivity and specificity of HBPM in diagnosis of masked HTN, ${ }^{8}$ there is available evidence from the Finn-Home study that HBPM is superior to 


\section{A}

Composite cardiovascular events: masked HTN versus normotension

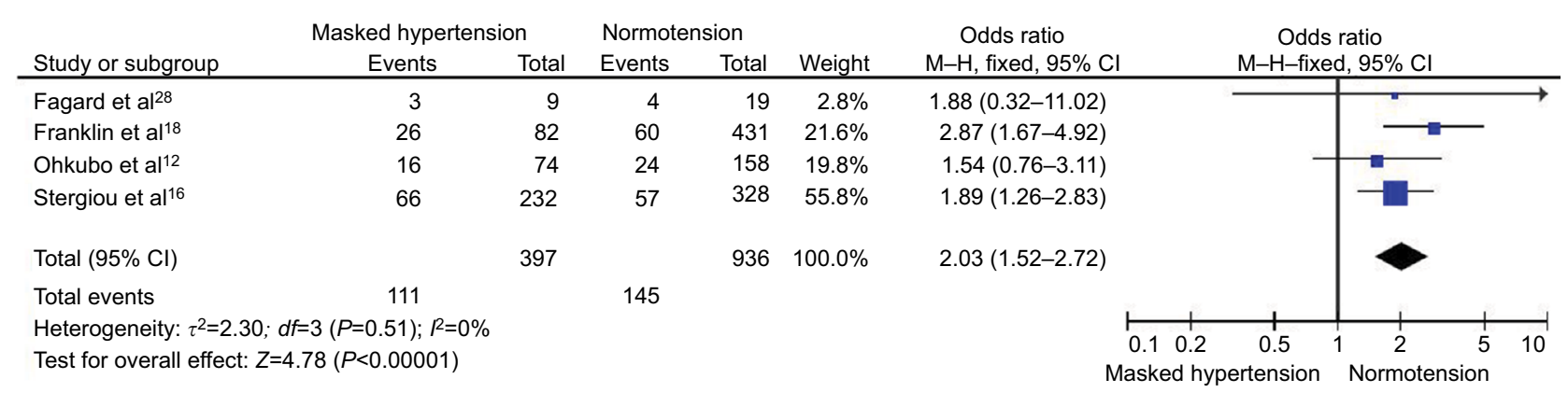

\section{B}

Mortality: masked HTN versus normotension

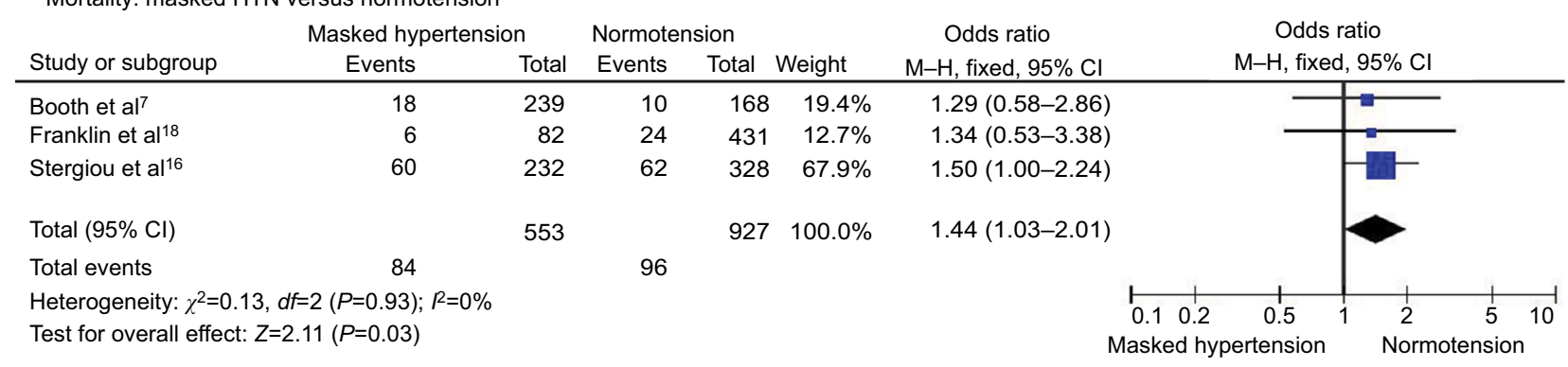

Figure 7 Masked HTN versus normotension - treated.

Notes: (A) Composite cardiovascular events. (B) All-cause mortality.

Abbreviations: $\mathrm{Cl}$, confidence interval; HTN, hypertension; $\mathrm{M}-\mathrm{H}$, Mantel-Haenszel.

\section{A}

Composite cardiovascular events: masked HTN versus sustained HTN

\begin{tabular}{|c|c|c|c|c|c|c|c|c|c|}
\hline \multirow[b]{2}{*}{ Study or subgroup } & \multicolumn{2}{|c|}{ Masked hypertension } & \multicolumn{2}{|c|}{ Sustained hypertension } & \multirow[b]{2}{*}{ Weight } & \multirow{2}{*}{$\begin{array}{c}\text { Odds ratio } \\
\mathrm{M}-\mathrm{H} \text {, random, } 95 \% \mathrm{Cl}\end{array}$} & \multirow{2}{*}{\multicolumn{2}{|c|}{$\begin{array}{l}\text { Odds ratio } \\
\mathrm{M}-\mathrm{H} \text {, random, } 95 \% \mathrm{Cl}\end{array}$}} & \\
\hline & Events & Total & Events & Total & & & & & \\
\hline Fagard et $\mathrm{al}^{28}$ & 3 & 9 & 18 & 51 & $7.0 \%$ & $0.92(0.20-4.11)$ & & & \\
\hline Franklin et al ${ }^{18}$ & 26 & 82 & 63 & 181 & $29.0 \%$ & $0.87(0.50-1.52)$ & & & \\
\hline Ohkubo et al ${ }^{12}$ & 16 & 74 & 26 & 96 & $21.9 \%$ & $0.74(0.36-1.52)$ & $\rightarrow$ & & \\
\hline Stergiou et al ${ }^{16}$ & 66 & 232 & 134 & 661 & $42.2 \%$ & $1.56(1.11-2.20)$ & & $\rightarrow-$ & \\
\hline Total $(95 \% \mathrm{Cl})$ & & 397 & & 989 & $100.0 \%$ & $1.08(0.71-1.65)$ & & & \\
\hline \multirow{2}{*}{\multicolumn{7}{|c|}{$\begin{array}{l}\text { Total events } \\
\text { Heterogeneity: } \tau^{2}=0.08 ; \chi^{2}=5.44, d f=3(P=0.14) ; P^{2}=45 \% \\
\text { Test for overall effect: } Z=0.36(P=0.72)\end{array}$}} & & & \\
\hline & & & & & & & $\begin{array}{ll}0.1 & 0.2\end{array}$ & 2 & 10 \\
\hline
\end{tabular}

B

Mortality: masked HTN versus sustained HTN

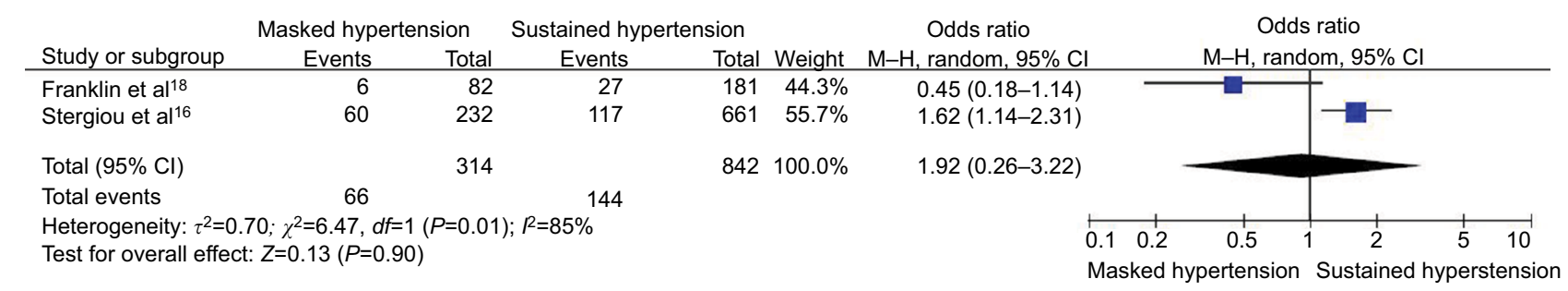

Figure 8 Masked HTN versus white-coat HTN - treated.

Notes: (A) Composite cardiovascular events. (B) All-cause mortality.

Abbreviations: $\mathrm{Cl}$, confidence interval; $\mathrm{HTN}$, hypertension; $\mathrm{M}-\mathrm{H}$, Mantel-Haenszel. 


\section{A}

Composite cardiovascular events: masked HTN versus white-coat HTN

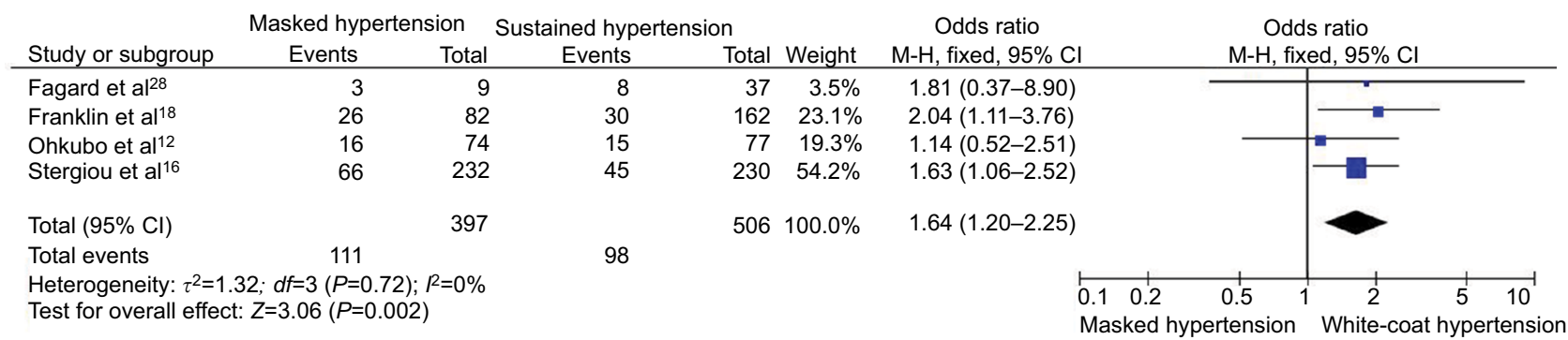

\section{B}

Mortality: masked HTN versus white-coat HTN

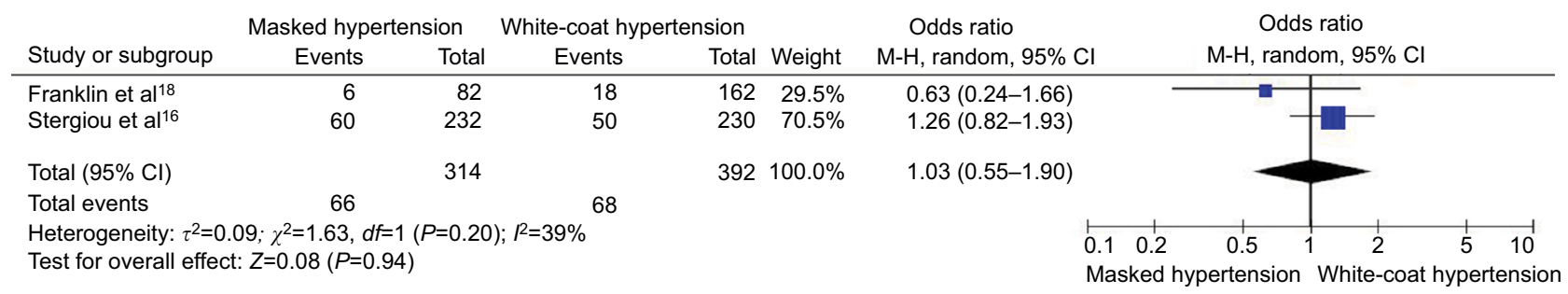

Figure 9 Masked HTN versus sustained HTN - treated.

Notes: (A) Composite cardiovascular events. (B) All-cause mortality.

Abbreviations: $\mathrm{Cl}$, confidence interval; HTN, hypertension; $\mathrm{M}-\mathrm{H}$, Mantel-Haenszel.

office BP in predicting cardiovascular risk and that HBPM should be used to screen for masked HTN in prehypertensive patients according to office BP. ${ }^{9}$ Therefore, a diagnosis of masked HTN can be based on HBPM and should be confirmed by ABPM before initiation of antihypertensive therapy.

Population cohort studies have suggested that among patients with masked HTN, one-third progresses to SH and one-third regresses to normotension in 5 years. ${ }^{10}$ Delayed diagnosis of masked HTN is related to high prevalence of hypertensive target organ damage in both treated and untreated patients with masked HTN. ${ }^{4}$ Work- and homerelated stress, smoking, age, sedentary lifestyle, and sleep disturbances have been identified as risk factors for masked HTN. ${ }^{11-14}$ It is suggested that masked HTN can be classified into masked daytime HTN and masked nocturnal HTN. ${ }^{15}$ Based on findings from the Jackson Heart study in blacks, nocturnal masked HTN is more frequent with the prevalence of 48.2 versus $28.2 \%$ prevalence of day-time masked HTN. The International Database of Home Blood Pressure in Relation to Cardiovascular Outcomes (IDHOCO) study demonstrated that masked HTN is more prevalent in treated hypertensive patients than in untreated individuals as antihypertensive treatment results in greater disproportional reduction of office BP than ABPM..$^{16,17}$ Noncompliance with medication, except just prior to office visits and morning peak levels of medications that coincide with office visits, may explain these observations. As emphasized in the International Database on Ambulatory Blood Pressure in Relation to Cardiovascular Outcomes (IDACO) study, antihypertensive treatment leads to a transformational change from $\mathrm{SH}$ to masked uncontrolled HTN and finally to sustained normotension. However, the risk of converted normotensives remains higher than that of normotensive individuals. ${ }^{18}$

Our findings are consistent with prior population-based studies such as the Dallas Heart Study and the Jackson Heart Study, which showed increased incidence of cardiovascular events in patients with masked HTN compared to patients with normotension, suggesting the malignant nature of masked HTN. ${ }^{6,7}$ Pooled analysis of 12 different populations from IDACO performed by Asayama et $\mathrm{al}^{32}$ showed similar findings. In contrast to the Jackson Heart Study, we found a significant increase in all-cause mortality in patients with masked HTN compared to patients with normotension. However, the Jackson Heart Study had small sample size with predominantly African American population. ${ }^{7}$ Our findings are consistent with large multiple population-based study performed by Stergiou et al, ${ }^{16}$ further potentiating the nature of masked HTN. Prior studies showed inconsistent results in cardiovascular outcomes between the patients with masked HTN and white-coat HTN. ${ }^{16,32}$ We found significant increase in composite cardiovascular events and all-cause mortality 
in patients with masked HTN compared to patients with white-coat HTN, which is similar to the study reported by Stergiou et al. ${ }^{16}$

Prevalence of masked HTN was found to be significantly high. Patients with masked HTN were $18 \%$ of population sample in the Dallas Heart Study. ${ }^{6}$ Similar results were seen in the pooled analysis of IDACO performed by Asayama et al. ${ }^{32}$ The Jackson Heart Study showed even higher prevalence patients with masked HTN. ${ }^{7}$ Furthermore, $>50 \%$ of these patient populations were untreated. ${ }^{6,7}$ The increased incidence of cardiovascular events and mortality in patients with masked HTN compared to patients with white-coat HTN are most likely due to the fact that the significant number of masked HTN patients being untreated. Subgroup analysis of patients treated with antihypertensives showed no significant difference in morality between the patients with masked HTN and white-coat HTN, suggesting that the patients with masked HTN who are successfully treated will have better outcomes. However, the cardiovascular events and mortality continued to be significantly high in patients with masked HTN compared to patients with normotension.

Based on current results, treating patients with masked HTN might prevent cardiovascular events and decrease the mortality. The two important issues to be addressed before treating the patients with masked HTN are how to diagnose masked HTN and what should be the target BP. Diagnosis can be made by ambulatory or home BP monitoring both in untreated and treated patients. ${ }^{33}$ However, identifying the patients to be screened is a more difficult task in untreated patients with masked HTN compared to treated patients with masked uncontrolled HTN. The main obstacle is diagnosing masked HTN in untreated population with normal office BP. ABPM and HBPM should have a complimentary role in the diagnosis and management of masked HTN. Patients with pre-HTN according to office BP, at high risk (diabetes, obstructive sleep apnea, and smoking), or evidence of target organ damage (chronic kidney disease and left ventricular hypertrophy) and normal office BP should be screened with HBPM. ${ }^{33-35}$ Nighttime and daytime BP recordings with ABPM may better define cardiovascular risk and guide treatment, as it prevents incomplete treatment of uncontrolled masked HTN.

Treating patients with masked HTN showed significant reductions in systolic BP; however, no significant difference was seen in end organ effects such as left ventricular mass index. ${ }^{36}$ Furthermore, there are no studies addressing the effect of treating masked HTN on clinic outcomes. Aggressive risk factors' modification such as obesity, diabetes, and sleep apnea would be an important step in preventing the adverse effects associated with masked HTN. ${ }^{33}$ Although HBPM rather than office BP measurements are more effective in guiding adjustments of antihypertensive medications to goal daytime BP, $\sim 25 \%$ of patients will be undertreated with this strategy due to the persistence of nocturnal HTN. Therefore, after reaching the daytime BP goal with HBPM, ABPM can establish whether escalation of antihypertensive treatment is required for the treatment of masked uncontrolled nocturnal HTN.

\section{Limitations}

There are some limitations to the interpretation of our data analysis. First, publication bias may still exist despite our best efforts to conduct a comprehensive search and despite the lack of statistical evidence for the existence of bias. Second, any meta-analysis based on the pooling of data from different cohorts with different inclusion criteria, different designs and populations, variable follow-up duration with differing attrition rates, and not being unified in definition and validation of endpoints in individual trials presents challenges. Moreover, our main endpoint, CVD events, was defined differently in most of the studies. Finally, the fact that some studies used ABPM and others used HBPM to define masked HTN may also increase the heterogeneity and affect the validity of the study.

The findings of our meta-analysis are in accordance with the findings of previous meta-analyses. ${ }^{37,38}$ Compared with previous works, our study is more comprehensive as it included slightly higher number of studies and analyzed not only cardiovascular events but also all-cause mortality as endpoints.

\section{Conclusion}

In this large meta-analysis of 14729 patients, the cardiovascular morbidity and mortality associated with masked HTN is higher than normotension and white-coat HTN but significantly lower than SH. Among treated patients, masked HTN and SH were associated with similar rates of cardiovascular events and mortality. Future studies should focus on the benefit of early screening and identification of patients with masked HTN and also evaluate BP treatment goals for these patients based on HBPM and ABPM.

\section{Author contributions}

All authors contributed toward data analysis, drafting and critically revising the paper, gave final approval of the version to be published, and agree to be accountable for all aspects of the work. 


\section{Disclosure}

The authors report no conflicts of interest in this work.

\section{References}

1. Egan BM, Zhao Y, Axon RN. US trends in prevalence, awareness, treatment, and control of hypertension, 1988-2008. JAMA. 2010;303(20): 2043-2050.

2. Wolf-Maier K, Cooper RS, Kramer H, et al. Hypertension treatment and control in five European countries, Canada, and the United States. Hypertension. 2004;43(1):10-17.

3. Mallion JM, Clerson P, Bobrie G, Genes N, Vaisse B, Chatellier G. Predictive factors for masked hypertension within a population of controlled hypertensives. $J$ Hypertens. 2006;24(12):2365-2370.

4. Hänninen MR, Niiranen TJ, Puukka PJ, Kesäniemi YA, Kähönen M, Jula AM. Target organ damage and masked hypertension in the general population: the Finn-Home study. J Hypertens. 2013;31(6):1136-1143.

5. Drawz PE, Alper AB, Anderson AH, et al; Chronic Renal Insufficiency Cohort Study Investigators. Masked hypertension and elevated nighttime blood pressure in CKD: prevalence and association with target organ damage. Clin J Am Soc Nephrol. 2016;11(4):642-652.

6. Tientcheu D, Ayers C, Das SR, et al. Target organ complications and cardiovascular events associated with masked hypertension and whitecoat hypertension: analysis from the Dallas Heart Study. $J$ Am Coll Cardiol. 2015;66(20):2159-2169.

7. Booth JN 3rd, Diaz KM, Seals SR, et al. Masked hypertension and cardiovascular disease events in a prospective cohort of blacks: the Jackson Heart Study. Hypertension. 2016;68(2):501-510.

8. Viera AJ, Hinderliter AL, Kshirsagar AV, Fine J, Dominik R. Reproducibility of masked hypertension in adults with untreated borderline office blood pressure: comparison of ambulatory and home monitoring. $\mathrm{Am}$ J Hypertens. 2010;23(11):1190-1197.

9. Niiranen TJ, Hänninen MR, Johansson J, Reunanen A, Jula AM. Homemeasured blood pressure is a stronger predictor of cardiovascular risk than office blood pressure: the Finn-Home study. Hypertension. 2010;55(6):1346-1351.

10. Trudel X, Milot A, Brisson C. Persistence and progression of masked hypertension: a 5-year prospective study. Int J Hypertens. 2013;2013:836387.

11. Landsbergis PA, Dobson M, Koutsouras G, Schnall P. Job strain and ambulatory blood pressure: a meta-analysis and systematic review. $\mathrm{Am}$ J Public Health. 2013;103(3):e61-e71.

12. Ohkubo T, Kikuya M, Metoki H, et al. Prognosis of "masked" hypertension and "white-coat" hypertension detected by 24 -h ambulatory blood pressure monitoring 10-year follow-up from the Ohasama study. $J \mathrm{Am}$ Coll Cardiol. 2005;46(3):508-515.

13. Cacciolati C, Tzourio C, Hanon O. Blood pressure variability in elderly persons with white-coat and masked hypertension compared to those with normotension and sustained hypertension. Am J Hypertens. 2013;26(3):367-372.

14. Mezick EJ, Hall M, Matthews KA. Sleep duration and ambulatory blood pressure in black and white adolescents. Hypertension. 2012;59(3):747-752.

15. Yano Y, Bakris GL. Recognition and management of masked hypertension: a review and novel approach. J Am Soc Hypertens. 2013;7(3):244-252.

16. Stergiou GS, Asayama K, Thijs L, et al; International Database on HOme blood pressure in relation to Cardiovascular Outcome (IDHOCO) Investigators. Prognosis of white-coat and masked hypertension: international database of home blood pressure in relation to cardiovascular outcome. Hypertension. 2014;63(4):675-682.

17. Schmieder RE, Schmidt ST, Riemer T, et al. Disproportional decrease in office blood pressure compared with 24-hour ambulatory blood pressure with antihypertensive treatment: dependency on pretreatment blood pressure levels. Hypertension. 2014;64(5):1067-1072.

18. Franklin SS, Thijs L, Li Y, et al; International Database on Ambulatory blood pressure in Relation to Cardiovascular Outcomes Investigators. Masked hypertension in diabetes mellitus: treatment implications for clinical practice. Hypertension. 2013;61(5):964-971.
19. Higgins JP, Altman DG, Gøtzsche PC, et al; Cochrane Bias Methods Group; Cochrane Statistical Methods Group. The cochrane collaboration's tool for assessing risk of bias in randomised trials. BMJ. 2011;343:d5928.

20. Higgins J, Green S. Cochrane Handbook for Systematic Reviews of Interventions Version 5.1.0. The Cochrane Collaboration; 2011. Available from: www.cochrane-handbook.org. Accessed November 28, 2017.

21. Moher D, Shamseer L, Clarke M, et al; PRISMA-P Group. Preferred reporting items for systematic review and meta-analysis protocols (PRISMA-P) 2015 statement. Syst Rev. 2015;4:1.

22. Higgins JP, Thompson SG, Deeks JJ, Altman DG. Measuring inconsistency in meta-analyses. BMJ. 2003;327(7414):557-560.

23. DerSimonian R, Laird N. Meta-analysis in clinical trials. Control Clin Trials. 1986;7(3):177-188.

24. Sterne JA, Egger M, Smith GD. Systematic reviews in health care: investigating and dealing with publication and other biases in metaanalysis. BMJ. 2001;323(7304):101-105.

25. Egger M, Davey Smith G, Schneider M, Minder C. Bias in meta-analysis detected by a simple, graphical test. BMJ. 1997;315(7109):629-634.

26. Duval S, Tweedie R. Trim and fill: a simple funnel-plot-based method of testing and adjusting for publication bias in meta-analysis. Biometrics. 2000;56(2):455-463.

27. Björklund K, Lind L, Zethelius B, Andrén B, Lithell H. Isolated ambulatory hypertension predicts cardiovascular morbidity in elderly men. Circulation. 2003;107(9):1297-1302.

28. Fagard RH, Van Den Broeke C, De Cort P. Prognostic significance of blood pressure measured in the office, at home and during ambulatory monitoring in older patients in general practice. J Hum Hypertens. 2005;19(10):801-807.

29. Mancia G, Facchetti R, Bombelli M, Grassi G, Sega R. Long-term risk of mortality associated with selective and combined elevation in office, home, and ambulatory blood pressure. Hypertension. 2006;47(5): 846-853.

30. Hansen TW, Jeppesen J, Rasmussen S, Ibsen H, Torp-Pedersen C. Ambulatory blood pressure monitoring and risk of cardiovascular disease: a population based study. Am J Hypertens. 2006;19(3):243-250.

31. Pierdomenico SD, Pannarale G, Rabbia F, et al. Prognostic relevance of masked hypertension in subjects with prehypertension. Am J Hypertens. 2008;21(8):879-883.

32. Asayama K, Thijs L, Li Y, et al; International Database on Ambulatory Blood Pressure in Relation to Cardiovascular Outcomes (IDACO) Investigators. Setting thresholds to varying blood pressure monitoring intervals differentially affects risk estimates associated with whitecoat and masked hypertension in the population. Hypertension. 2014;64(5):935-942.

33. Peacock J, Diaz KM, Viera AJ, Schwartz JE, Shimbo D. Unmasking masked hypertension: prevalence, clinical implications, diagnosis, correlates and future directions. J Hum Hypertens. 2014;28(9):521-528.

34. Shimbo D, Newman JD, Schwartz JE. Masked hypertension and prehypertension: diagnostic overlap and interrelationships with left ventricular mass: the Masked Hypertension Study. Am J Hypertens. 2012;25(6):664-671.

35. Hänninen MR, Niiranen TJ, Puukka PJ, Mattila AK, Jula AM. Determinants of masked hypertension in the general population: the Finn-Home study. J Hypertens. 2011;29(10):1880-1888.

36. Hare JL, Sharman JE, Leano R, Jenkins C, Wright L, Marwick TH. Impact of spironolactone on vascular, myocardial, and functional parameters in untreated patients with a hypertensive response to exercise. $\mathrm{Am}$ J Hypertens. 2013;26(5):691-699.

37. Fagard RH, Cornelissen VA. Incidence of cardiovascular events in whitecoat, masked and sustained hypertension versus true normotension: a meta-analysis. J Hypertens. 2007;25(11):2193-2198.

38. Pierdomenico SD, Cuccurullo F. Prognostic value of white-coat and masked hypertension diagnosed by ambulatory monitoring in initially untreated subjects: an updated meta-analysis. Am J Hypertens. 2011;24(1):52-58. 


\section{Publish your work in this journal}

Integrated Blood Pressure Control is an international, peer-reviewed open-access journal focusing on the integrated approach to managing hypertension and risk reduction. Treating the patient and comorbidities together with diet and lifestyle modification and optimizing healthcare resources through a multidisciplinary team approach constitute key features of the journal. This journal is indexed on American Chemical Society's Chemical Abstracts Service (CAS). The manuscript management system is completely online and includes a very quick and fair peerreview system, which is all easy to use. Visit http://www.dovepress.com/ testimonials.php to read real quotes from published authors.

Submit your manuscript here: https://www.dovepress.com/integrated-blood-pressure-control-journal 